\title{
Catalogue of Pavements with Recycled Aggregates from Construction and Demolition Waste ${ }^{\dagger}$
}

\author{
Auxi Barbudo *, José Ramón Jiménez, Jesús Ayuso, Adela Pérez Galvín and Francisco Agrela \\ Construction Engineering Area, University of Cordoba, 14071 Cordoba, Spain; jrjimenez@uco.es (J.R.J.); \\ ir1ayuje@uco.es (J.A.); apgalvin@uco.es (A.P.G.); fagrela@uco.es (F.A.) \\ * Correspondence: abarbudo@uco.es; Tel.: +34-957-218-547 \\ † Presented at Environment, Green Technology and Engineering International Conference (EGTEIC 2018), \\ Caceres, Spain, 18-20 June 2018.
}

Published: 18 October 2018

\begin{abstract}
Construction and Demolition Waste come from debris generated during construction, renovation and demolition of buildings, roads, and bridges. Recycling and reuse are essential in terms of sustainability, mainly from an environmental point of view. Although the recommendation of the use of these recycled aggregates is currently included in some technical specifications, its use is still not widespread due mainly to the lack of knowledge on their technical application. This work is a compilation of the recommendations proposed in the "Catalogue of road pavements with recycled aggregates", supported by the construction of experimental stretches. It proposes different structural sections for road pavements by using recycled aggregates.
\end{abstract}

Keywords: construction and demolition waste; recycled aggregates; catalogue; pavements

\section{Introduction}

Construction and Demolition Waste (CDW) is a priority waste stream in the European Union. Currently, the production of CDW in Spain is around 20 million tons (430 kg/habitant.year). The Waste Framework Directive requires Member States to recycle 70\% of CDW before 2020. Recycled aggregates (RA) which are obtained from the CDW conveniently treated in recycling plants, can be used in civil engineering and building works. The recycling rate in Spain is approximately $40 \%$, very far from recycling rates in other countries such as the Netherlands, Denmark and Germany that exceed the recycling rate of $80 \%$.

The RA have different physical-mechanical and chemical properties from natural aggregates (NA). These have a lower density, a higher water absorption, a lower resistance to fragmentation and a higher content of sulfur compounds and soluble salts [1-3]. However, these properties should not limit its use while the infrastructure in which they use has adequate functional and structural characteristics.

Habitually, RA are rejected because they do not meet the technical specifications required for NA in regulations such as the Spanish General Technical Specifications for Road Construction (PG3) [4] or the Structural Concrete Instruction (EHE-08) [5]. It should be noted that the PG-3 is applicable to the Road Network of the Spanish State and that the EHE-08 constitutes the framework in which the requirements considered in the design and manufacturing of concrete structures are established. However, there are other uses with lower requirements, such as roads with low traffic intensity [6,7], bike lines [8], urban and pedestrian roads, unpaved rural roads [9-11] where RA provide similar functional and structural characteristics to the of NA.

Hence, to increase the recycling rate, it is necessary to develop a specific technical regulation and new uses for RA. In this sense, the University of Córdoba (Construction Engineering Area), together 
with the Regional Ministry of Public Works and Housing of the Regional Government of Andalusia, recycling companies (AGRECA) and companies in the construction sector (CEMOSA) have developed a "Catalogue of road pavements with recycled aggregates (CRA-2017)" [12]. This technical document proposes constructive solutions designed with RA and supported by the construction of experimental sections. The CRA-2017 is the first document of these characteristics in Spain.

\section{Area of Application}

The scope of application of this Catalogue are the pavements sections, where RA is currently used, but without a specific technical regulation for it, such as:

- Pavements roadworks with traffic intensity lower than T2 ( $<800$ heavy vehicles/day).

- Pavements of rural roads.

- Steel and pedestrian routes.

- Pavements of cyclist's routes.

- Drainage works and ditches.

This paper presents the technical requirements of the materials, the project criteria and constructive solutions with RA proposed for road and rural roads.

\section{Characteristics Required of Materials to Pavements}

Figure 1 shows the types of pavement structural sections recommended for using RA (their use on rigid pavements has not been considered, nor on roads with traffic levels greater than T2). The recycled materials for the pavement can be classified as well-graded recycled concrete aggregate (ZARHor), well-graded mixed recycled aggregate (ZARM I and ZARM II), recycled cement-soil (SCR) and the roller compacted recycled concrete (HCR). Table 1 shows the classification of recycled well-graded granular materials according to their composition.
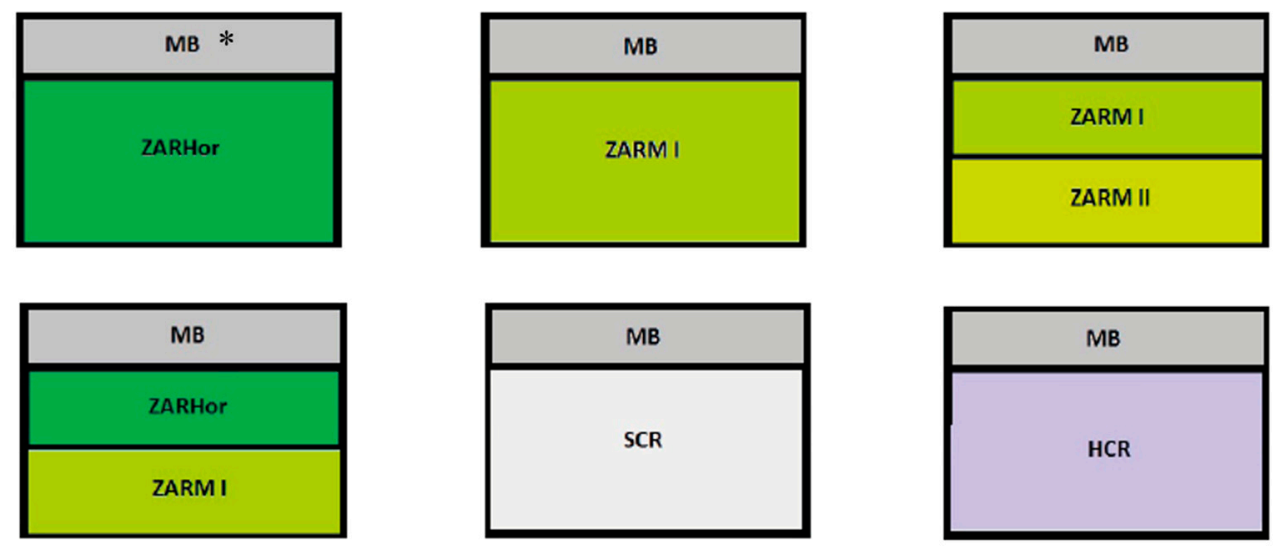

Figure 1. Types of structural sections recommended in the catalogue. ${ }^{*} \mathrm{MB}=$ Bituminous mixture.

Table 1. Classification of recycled well-graded granular materials according to composition test.

\begin{tabular}{ccccc}
\hline & & ZARHor & ZARM I & ZARM II \\
\hline & $\mathrm{Rc}+\mathrm{Ru}+\mathrm{Ra}$ & - & $\geq 70 \%$ & $\geq 70 \%$ \\
& $\mathrm{Rc}+\mathrm{Ru}$ & $\geq 90 \%$ & $\geq 55 \%$ & $\geq 55 \%$ \\
Composition & $\mathrm{Rc}$ & - & - & - \\
EN 933-11 & $\mathrm{Ra}$ & - & - & - \\
& $\mathrm{Rb}$ & - & - & - \\
& $\mathrm{X}$ & & $<1 \%$ & $<2 \%$ \\
& $\mathrm{FL}$ & $<1 \mathrm{~cm}^{3} / \mathrm{kg}$ & $<2 \mathrm{~cm}^{3} / \mathrm{kg}$ \\
\hline
\end{tabular}

Footnote: $\mathrm{Rc}=$ concrete particles; $\mathrm{Ru}=$ aggregates not bonded with cement; $\mathrm{Ra}=$ asphalt particles; $\mathrm{Rb}$ = ceramic particles; $X=$ impurities (woods, plastics, plasters, glass, etc.); FL: floating particles. 
In order to analyze the failure of the pavement layers, it has been considered as critical parameters the following:

- $\quad$ The maximum tensile stress ( $\sigma r)$ in cement-treated materials.

- The unitary vertical deformation in the upper face $(\varepsilon z)$ in the subgrades.

Each material has been considered separately by selecting the stress or critical deformation given by the response model, which is introduced in the fatigue law, which provides the admissible number of load applications (N). The lower value of $\mathrm{N}$ among those obtained for all the layers of the firm will represent the number of load applications that could cause the structure to collapse the pavement by fatigue. Table 2 shows the characteristics of the materials and fatigue laws used for each material.

Table 2. Characteristics of the materials and fatigue laws.

\begin{tabular}{cccc}
\hline Recycled Material-Pavements & Modulus & Poisson's Coefficient & Fatigue \\
\hline ZARHor & $500 \mathrm{MPa}$ & 0.35 & \\
ZARM I & $400 \mathrm{MPa}$ & 0.35 & \multirow{2}{*}{0.35} \\
ZARM II & $300 \mathrm{MPa}$ & \multirow{2}{*}{0.25} & $\sigma \mathrm{r}=0.43(1-0.065 \operatorname{logN})$ \\
SCR-3 & \multirow{2}{*}{$8000 \mathrm{MPa}$} & $\sigma \mathrm{r}=0.72(1-0.065 \operatorname{logN})$ \\
SCR-4 & & 0.25 & $\sigma \mathrm{r}=1.03(1-0.065 \operatorname{logN})$ \\
\hline HCR & $13,000 \mathrm{MPa}$ &
\end{tabular}

\section{Typical Sections on Roads Pavements}

The catalogue shows solutions for the different categories of subgrade (E1: low category subgrade, E2: medium category subgrade and E3: high category subgrade) (Table 3) and different T2 traffic categories ( $\geq 200$ and $<800$ heavy vehicles/day); T3A ( $\geq 100$ and $<200$ heavy vehicles/day), T3B ( $\geq 50$ and $<100$ heavy vehicles/day), T4A ( $\geq 25$ and $<50$ heavy vehicles/day), T4B ( $<25$ heavy vehicles/ day).

Table 3. Categories of the subgrade.

\begin{tabular}{ccc}
\hline Category of the Subgrade & Equivalent Module, Ee (MPa) & Valid Categories of Project Traffic \\
\hline Low & $\geq 60$ & T4 \\
Medium & $\geq 100$ & T3 and T4 \\
High & $\geq 160$ & T00 to T2 \\
\hline
\end{tabular}

For the calculation of the pavement structure layers, the multilayer elastic response model developed by Burmister has been used to obtain the response in stresses and deformations in the layers of the road subject to stresses.

\section{Conclusions}

This work is extracted from "Catalogue of pavements with recycled CDW aggregates (CRA)", redacted with the aim of promoting the use of recycled materials in roads in the framework of a sustainable perspective and immersed in the future policies of the European Union.

Author Contributions: All authors have actively worked on the project and developed as co-authors a "CRA2017" which this communication is extracted. J.R.J. and A.B. have worked in the preparation of the communication and write the full paper, J.A. have reviewed this work and A.P.G. reviewed the language.

Acknowledgments: The authors would like to thank the ERDF of the European Union and the Public Works Agency and Regional Ministry of Public Works and Housing of the Regional Government of Andalusia for financial support via the project "Applications of recycled aggregates from construction and demolition waste (CDW) for the sustainable development of road infrastructure in central areas of Andalusia" from the "20072013 ERDF Operational Program for Andalusia. Thanks also to Association of Construction Waste Management Companies and Demolition of Andalusia (AGRECA) and the Center for Studies of Materials and Works Control SA (CEMOSA) (no funds for covering the costs to publish in open Access). 
Conflicts of Interest: The authors declare no conflict of interest. The funding sponsors that have participated in the design of the study (Regional Ministry of Public Works and Housing of the Regional Government of Andalusia) have been already indicated in the Acknowledgments. In any case, the funding sponsors don't have interest or benefit on the results obtained.

\section{References}

1. Agrela, F.; De Juan, M.S.; Ayuso, J.; Giraldes, V.L.; Jiménez, J.R. Limiting properties in the characterisation of mixed recycled aggregates for use in the manufacture of concrete. Constr. Build. Mater. 2011, 25, 39503955.

2. Barbudo, A.; Agrela, F.; Ayuso, J.; Jiménez, J.R.; Poon, C.S. Statistical analysis of recycled aggregates derived from different sources for sub-base applications. Constr. Build. Mater. 2012, 28, 129-138.

3. Jiménez, J.R. Recycled aggregates (RAs) for roads. In Handbook of Recycled Concrete and Demolition Waste; Woodhead Publishing Limited: Cambridge, UK, 2013; pp. 351-377.

4. Spanish General Technical Specifications for Road Construction (PG3); Ministry of Development, Government of Spain: Madrid, Spain, 2004. (In Spanish)

5. Structural Concrete Instruction (EHE-08); Ministry of Development: Madrid, Spain, 2008.

6. Agrela, F.; Barbudo, A.; Ramírez, A.; Ayuso, J.; Carvajal, M.D.; Jiménez, J.R. Construction of road sections using mixed recycled aggregates treated with cement in Malaga, Spain. Resour. Conserv. Recycl. 2012, 58, 98-106.

7. Tavira, J.; Jiménez, J.R.; Ayuso, J.; López-Uceda, A.; Ledesma, E.F. Recycling screening waste and recycled mixed aggregates from construction and demolition waste in paved bike lanes. J. Clean. Prod. 2018, 190, 211-220.

8. Tavira, J.; Jiménez, J.R.; Ayuso, J.; Sierra, M.J.; Ledesma, E.F. Functional and structural parameters of a paved road section constructed with mixed recycled aggregates from non-selected construction and demolition waste with excavation soil. Constr. Build. Mater. 2018, 164, 57-69.

9. Jiménez, J.R.; Ayuso, J.; Galvín, A.P.; López, M.; Agrela, F. Use of mixed recycled aggregates with a low embodied energy from non-selected CDW in unpaved rural roads. Constr. Build. Mater. 2012, 34, $34-43$.

10. Jimenez, J.R.; Ayuso, J.; Agrela, F.; López, M.; Galvín, A.P. Utilisation of unbound recycled aggregates from selected CDW in unpaved rural roads. Resour. Conserv. Recycl. 2012, 58, 88-97.

11. Del Rey, I.; Ayuso, J.; Galvín, A.P.; Jiménez, J.R.; Barbudo, A. Feasibility of using unbound mixed recycled aggregates from CDW over expansive clay subgrade in unpaved rural roads. Materials, 2016, 9, 931.

12. Public Works Agency and Regional Ministry of Public Works and Housing of the Regional Government of Andalusia. Catalogue of Road Pavements and Work Units with Recycled Aggregates; UCOPress, Editorial Universidad de Córdoba: Cordoba, Spain, 2017. Available online: http://www.aridosrcdandalucia.es/rcd/ catalogo-de-firmes/ (accessed on 30 May 2018). (In Spanish) 